\title{
Scanning capacitance microscopy of nanostructures
}

\author{
H. E. Ruda and A. Shik \\ Centre for Advanced Nanotechnology, University of Toronto, Toronto M5S 3E4, Canada
}

(Received 10 May 2004; revised manuscript received 10 November 2004; published 18 February 2005)

\begin{abstract}
A theory is developed for scanning capacitance microscopy (SCM) of samples containing quantum wells, nanowires, and nanodots. The observed SCM image does not reproduce the geometric characteristics of the system but can be used for extracting some important information. For quantum wells and nanowire arrays, SCM gives an opportunity to determine the doping level of nanostructures. For individual nanowires and dots, the positions and amplitude of SCM maxima give, respectively, the in-plane location and the depth of the objects, and the pattern shape is extremely sensitive to the nanowire orientation.
\end{abstract}

DOI: 10.1103/PhysRevB.71.075316

PACS number(s): 73.63.Rt, 41.20.Cv, 84.37.+q

\section{INTRODUCTION}

Scanning capacitance microscopy (SCM) is a powerful characterization method, which can be used for investigation of the doping profile in semiconductors. ${ }^{1}$ Theoretical analysis of the method ${ }^{2}$ shows that its spatial resolution is essentially worse than that of other related methods of scanning microscopy, such as scanning tunneling or atomic force microscopy, and is determined by the screening radius in a given sample, $r_{s}$. In the present work, we discuss considerations for using SCM for characterization of nanostructures with a typical size less than $r_{s}$. Although SCM profiling in this case does not directly reproduce the composition and/or doping profile of a nanostructure, this does not exclude the possibility of obtaining interesting and important information on nanostructures. However, the character of this information can be different for different types of nanostructures and should be elucidated for each type separately.

As in our previous work, ${ }^{2}$ we assume that the SCM tip has a parabolic geometry with radius of curvature, $R$, and placed at a distance $d$ from the sample surface $z=0$ (Fig. 1).

\section{QUANTUM WELLS AND HETEROJUNCTIONS}

Consider a semiconductor structure containing a twodimensional electron gas (selectively doped heterojunction or quantum well) with areal density $N$ at a depth $a$ from the surface [Fig. 1(a)]. Applying a voltage $V$ to the tip causes a redistribution of the electron density $n(\rho)$ which, in turn, results in a circularly symmetric surface charge $e[n(\rho)-N]$ and potential distribution $\varphi(\rho, z)$. If the depth $a$ is sufficiently small, then we can assume $(\partial \varphi / \partial z)(\rho,-a)$ $\simeq(\partial \varphi / \partial z)\left(\rho, 0^{-}\right)=(1 / \varepsilon)(\partial \varphi / \partial z)\left(\rho, 0^{+}\right) \quad$ and $\varphi(\rho,-a)$ $\simeq \varphi(\rho, 0)-a(\partial \varphi / \partial z)\left(\rho, 0^{-}\right)$where $\varepsilon$ is the sample dielectric constant. Using the relationship between $\varphi(\rho, 0)$ and $(\partial \varphi / \partial z)\left(\rho, 0^{+}\right)$at the sample surface derived in Ref. 2 for a parabolic tip, we obtain the connection between potential and normal electric field in the plane of the two-dimensional electron gas:

$$
\begin{gathered}
\frac{\varepsilon}{2} \ln \left(\frac{\sqrt{d+R}+\sqrt{d}}{\sqrt{d+R}-\sqrt{d}}\right) \sqrt{\rho^{2}+d^{2}+R d} \frac{\partial \varphi}{\partial z}(\rho,-a) \\
=V-\varphi(\rho,-a)-a \frac{\partial \varphi}{\partial z}(\rho,-a)
\end{gathered}
$$

If the applied bias $V$ is positive or not of a large negative value, screening of the tip potential in the electron layer has a linear character so that for degenerate two-dimensional electrons gas we have $\varphi(\rho,-a)=\left(\varepsilon a_{B} / 4\right)(\partial \varphi / \partial z)(\rho,-a)$ where $a_{B}=\hbar^{2} /\left(m e^{2}\right)$ ( $m$ is the electron effective mass) is the effective Bohr radius. By using this expression, we immediately obtain from Eq. (1) $(\partial \varphi / \partial z)(\rho,-a)$ and hence the surface charge density

$$
\begin{aligned}
\sigma_{0}(\rho) & =-\frac{\varepsilon}{4 \pi} \frac{\partial \varphi}{\partial z}(\rho,-a) \\
& =\frac{-V}{2 \pi\left[\ln \left(\frac{\sqrt{d+R}+\sqrt{d}}{\sqrt{d+R}-\sqrt{d}}\right) \sqrt{\rho^{2}+d^{2}+R d}+\frac{2 a}{\varepsilon}+\frac{a_{B}}{2}\right]}
\end{aligned}
$$

The terms in denominator containing $a$ and $a_{B}$ describe contributions to the total capacity caused by the capacity of capping layer $a$ and by the quantum capacity of the twodimensional electron gas. ${ }^{3}$ Note that our assumption of a two-dimensional and degenerate character of the electron gas is not crucial, and Eq. (2) can be generalized to any other situation by replacing $a_{B} / 2$ with the corresponding screening length $r_{s}$.

Integration of Eq. (2) this over $\rho$ gives the capacity $C$, which is similar to that obtained in Ref. 2 for a bulk metallic sample and is independent of $V$. Nonzero values of $d C / d V$ can be observed at large depleting (negative) bias exceeding some critical value $V_{0}$, determined below, when the region directly below the tip becomes completely depleted.

For a degenerate two-dimensional electron gas, the boundary of complete depletion $\rho_{0}$ is the point where the band edge crosses the Fermi level $\pi \hbar^{2} N / m$ and the boundary condition for potential changes its character:

$$
\begin{gathered}
\frac{\partial \varphi}{\partial z}(\rho,-a)=-\frac{4 \pi e N}{\varepsilon}, \quad \rho \leqslant \rho_{0}, \\
\frac{\partial \varphi}{\partial z}(\rho,-a)=\frac{2}{\varepsilon r_{s}} \varphi(\rho,-a), \quad \rho \geqslant \rho_{0} .
\end{gathered}
$$



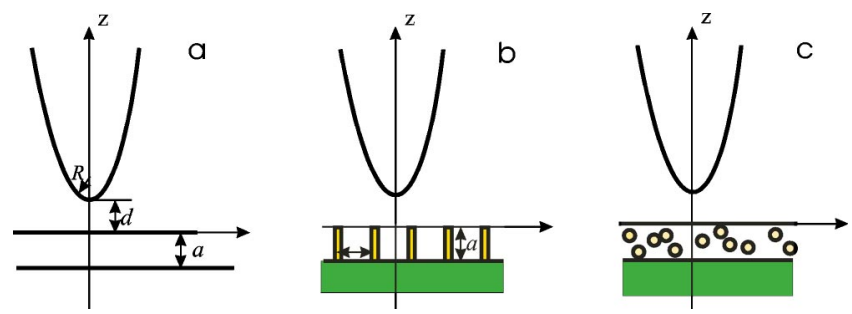

FIG. 1. Schematic illustration of SCM measurements in nanostructures: (a) single quanum well parallel to the surface, (b) array of nanowires on heavily doped substrate, and (c) system of quantum dots. Heavily doped substrate (the back contact) is shown by dark filling, doped nanowires by light filling.

Substitution of the first equation of Eqs. (3) into Eq. (1) gives the potential profile at $\rho<\rho_{0}$, as well as the values of $V_{0}$ and $\rho_{0}$ :

$$
\begin{gathered}
V_{0}=2 \pi e N\left[\ln \left(\frac{\sqrt{d+R}+\sqrt{d}}{\sqrt{d+R}-\sqrt{d}}\right) \sqrt{d^{2}+R d}+\frac{2 a}{\varepsilon}+r_{s}\right], \quad(4) \\
\rho_{0}^{2}=\left[\frac{|V|-V_{0}}{2 \pi e N \ln \left(\frac{\sqrt{d+R}+\sqrt{d}}{\sqrt{d+R}-\sqrt{d}}\right)}\right]^{2}+\frac{\left(|V|-V_{0}\right) \sqrt{d^{2}+R d}}{\pi e N \ln \left(\frac{\sqrt{d+R}+\sqrt{d}}{\sqrt{d+R}-\sqrt{d}}\right)} .
\end{gathered}
$$

Now one can calculate $d C / d V$, the main characteristic of $\mathrm{SCM}$. Since for the charge distribution, Eq. (2), $d C / d V=0$, a nonzero contribution arises only from the region $\rho<\rho_{0}$ where $\sigma_{0}(\rho)$ should be replaced by $e N$. Using Eqs. (2), (4), and (5) gives for, $V<-V_{0}$,

$$
\begin{aligned}
\frac{d C}{d V} & =2 \pi \frac{d^{2}}{d V^{2}} \int_{0}^{\rho_{0}}\left[\sigma_{0}(\rho)-e N\right] \rho d \rho \\
& =\frac{2 \sqrt{d^{2}+R d}}{V_{0} \ln \left(\frac{\sqrt{d+R}+\sqrt{d}}{\sqrt{d+R}-\sqrt{d}}\right)}=\operatorname{const}(V) .
\end{aligned}
$$

For $V>-V_{0}$ screening is linear and $d C / d V=0$. The abrupt drop in $d C / d V$ at $V=-V_{0}$ results from the assumption of complete depletion and should be thermally broadened in the bias interval $\sim k T / e$. The horizontal $\left(-V_{0}\right)$ and vertical [Eq. (6)] coordinates of the step at $d C / d V$ vs $V$ permit an independent determination of the doping level of the well $N$ [see Eq. (4)] and the tip parameter $\sqrt{d^{2}+R d}$. We should emphasize that Eq. (6) does not contain $a$ and $r_{s}$ (the only parameter depending on the density of states) and hence is valid for both thin classical films and two-dimensional quantum wells, which is due to the fact that the two corresponding components of the total capacity are voltage independent and give no contribution to $d C / d V$.

\section{ARRAYS OF NANOWIRES}

Consider another type of nanostructure: namely, nanowires. Individual electrically isolated wires will be analyzed in the next section while here we investigate wires having electical contact with a conducting substrate used as the second electrode in SCM measurements. Such structures are usually fabricated either using electrochemically etched $\mathrm{Al}_{2} \mathrm{O}_{3}$ films with a periodic system of cylindrical voids filled by semiconductor ${ }^{4,5}$ or as an array of free-standing wires grown from gold nanoislands. ${ }^{6,7}$ If the upper contact has a large area so that many wires are connected in parallel, the system has a number of interesting features in its macroscopic conductivity $^{8}$ and capacity. ${ }^{9}$ We consider the behavior of such a system in SCM measurements [Fig. 1(b)].

We use the perturbation approach assuming that individual wires do not seriously influence the potential distribution between the tip and conducting substrate calculated in the Appendix. For a negative applied voltage $V$, the parts of wires where $|e \varphi|>k T$ are depleted and their charge determines $C_{1}$ - the addition to capacity, which, in contrast to $C_{0}$, depends on $V$ and on the tip position and is measurable in modulation SCM experiments. Calculations become easier if we note that the potential, Eq. (17), deep in the sample, at $z$ approaching $-a$, is linear in $z$ and for $\sqrt{d^{2}+R d} \ll a$ can be written as

$$
\varphi(\rho, z) \simeq \frac{2 V(z+a)}{\varepsilon \ln \left(\frac{\sqrt{d+R}+\sqrt{d}}{\sqrt{d+R}-\sqrt{d}}\right)} \int_{0}^{\infty} \frac{\mathrm{J}_{0}(\lambda \rho) d \lambda}{\cosh (\lambda a)} .
$$

Using Eq. (7) one can determine $C_{1}$ caused by a single nanowire placed at the distance $\rho_{0}$ from the tip axis $(\rho=0)$. If $\nu$ is the doping level of nanowires (per unit length), then the total wire charge $Q=e \nu\left\{a-\int_{-a}^{0} \exp \left[e \varphi\left(\rho_{0}, z\right) / k T\right] d z\right\}$ and

$$
\begin{aligned}
C_{1}= & -\frac{d Q}{d V} \\
= & \frac{e \nu a}{V}\left[-\frac{k T}{e V f\left(\rho_{0}\right)} \exp \left(\frac{e V f\left(\rho_{0}\right)}{k T}\right)\right. \\
& \left.+\frac{k T}{e V f\left(\rho_{0}\right)}+\exp \left(\frac{e V f\left(\rho_{0}\right)}{k T}\right)\right],
\end{aligned}
$$

where

$$
f\left(\rho_{0}\right)=\frac{2}{\varepsilon \ln \left(\frac{\sqrt{d+R}+\sqrt{d}}{\sqrt{d+R}-\sqrt{d}}\right)} \int_{0}^{\infty} \frac{\mathrm{J}_{0}\left(t \rho_{0} / a\right) d t}{\cosh (t)}
$$

is a decreasing function of $\rho_{0}$. We restrict ourselves to the depleting bias $(V<0)$ since in the case of accumulation most carriers determining $C_{1}$ are concentrated near the nanowire end where the linear approximation, Eq. (7), fails.

According to Eq. (8), $C_{1}$ as a function of $f\left(\rho_{0}\right)$ has a maximum at $e|V| f\left(\rho_{0}\right) / k T \simeq 1.8$. As a result, the assumption of $C_{1}$ being maximal for a nanowire located exactly below the tip is correct only at sufficiently low bias,

$$
e|V|<1.4 \varepsilon k T \ln \left(\frac{\sqrt{d+R}+\sqrt{d}}{\sqrt{d+R}-\sqrt{d}}\right) .
$$

The explanation is evident: at high $|V|$ the nanowire just beneath the tip may become completely depleted up to the 
highly conducting substrate with $Q=\operatorname{const}(V)$, while more distant, not completely depleted, nanowires contribute to $C_{1}$ more noticeably. Practically this means that for a depletion bias exceeding the critical value, Eq. (9), the spatial SCM pattern "inverts" so that nanowire locations become the points of minimal, rather than maximal, local capacitance and the total SCM image of a wire has an annular shape. As to the voltage dependence of $C_{1}$ on $|V|$, it is monotonically decreasing for any $\rho_{0}$ (at high bias $C_{1} \sim V^{-2}$ ), since the situation approaches gradually the case of complete depletion when the wire charge becomes voltage independent. At small $V, C_{1}$ acquires a constant value, at the nanowire axis $\left(\rho_{0}\right.$ $=0)$ equal to

$$
C_{1}=\frac{\pi e^{2} \nu a}{\varepsilon \ln \left(\frac{\sqrt{d+R}+\sqrt{d}}{\sqrt{d+R}-\sqrt{d}}\right)} .
$$

Equation (10) is proportional to the nanowire doping level $\nu$ and can be used for its determination. If the logarithm in the formula is unknown, it can be found from Eq. (9) by measuring the bias for which the above-mentioned pattern "inversion" occurs.

The potential, Eq. (7), drops in the $\rho$ direction at the distance of order $a$. Thus, if one considers not a single wire but a wire array with the interwire distance $\delta<a, C_{1}$ is determined by a large, $\sim(a / \delta)^{2}$, number of wires. As a result, spatial variations of the measured capacity and its derivatives will be small. [This agrees with the general statement of SCM spatial resolution to be determined by the sample screening radius $r_{s}^{2}$, recalling that the role of $r_{s}$ in an array of parallel wires is played by the interwire distance $\delta$ (Ref. 10).] Moreover, the linear capacity $C$ obtained from a summation of $C_{1}$ over all wires diverges similarly to the capacity in macroscopic samples ${ }^{2}$ and physically interesting information can be obtained only from $d C / d V$, which varies from point to point with relative amplitude $\sim(\delta / a)^{2}$. Note that $d C_{1} / d V$, contrary to $C_{1}$, depends on $\rho_{0}$ monotonically and thus $d C / d V$ for any $V$ is maximal for the tip position at a wire axis. As discussed, at small $\delta / a$ the spatial contrast of $d C / d V$ becomes very small, which may prevent an observer from distinguishing separate wires.

\section{INDIVIDUAL NANOWIRES AND DOTS}

\section{A. General approach}

Thus far, we have considered nanostructures, which from the electrical point of view represented a single-connected conducting entity serving as the second electrode having potential $-V$ relative to the tip. For a system of electrically isolated individual nanowires or dots the approach should be different. In this case, one cannot contact each nanoelement individually and must create an additional electrode ( e.g., by a metal film at the backside of the sample [Fig. 1(c)]). In this situation, the applied bias does not charge the nanoelement but only polarizes it. Due to the small size of a nanodot or nanowire, this polarization does not noticeably change the distribution of electric field between the tip and back elec- trode, which allows us to use the perturbation approach. This approach includes the following steps: (i) determination of the electric field profile between the tip and backside electrode and (ii) calculation of the polarizability $\alpha_{i j}$ of nanodots (nanowires) in the external electric field $\mathbf{E}$ and the change of electrostatic energy $\Delta W=\sum_{i, j} \alpha_{i j} E_{i} E_{j} / 2$ caused by nanostructure polarization.

The first step is made in the Appendix. By differentiating Eq. (17), we immediately obtain the components of electric field inside the sample:

$$
\begin{aligned}
E_{\rho}(\rho, z)= & \frac{-2 V}{\varepsilon \ln \left(\frac{\sqrt{d+R}+\sqrt{d})}{\sqrt{d+R}-\sqrt{d}}\right)} \int_{0}^{\infty} \frac{\sinh [\lambda(z+a)]}{\cosh (\lambda a)} \mathrm{J}_{1}(\lambda \rho) \\
& \times \exp \left(-\lambda \sqrt{d^{2}+R d}\right) d \lambda, \\
E_{z}(\rho, z)= & \frac{2 V}{\varepsilon \ln \left(\frac{\sqrt{d+R}+\sqrt{d}}{\sqrt{d+R}-\sqrt{d}}\right)} \int_{0}^{\infty} \frac{\cosh [\lambda(z+a)]}{\cosh (\lambda a)} \mathrm{J}_{0}(\lambda \rho) \\
& \times \exp \left(-\lambda \sqrt{d^{2}+R d}\right) d \lambda .
\end{aligned}
$$

Comparison of these two formulas shows that for all values of $z, d, R$, and $a$ the ratio $\left|E \rho / E_{z}\right|$ tends to zero at $\rho \rightarrow 0$ and $\rho \rightarrow \infty$, having a maximum at $\rho \sim a$. The value in the maximum approaches 1 at very small $z, d$, and $R$ and is essentially less far from the tip.

Since all components $E_{i}$ are proportional to the applied voltage $V$ and depend on the coordinates $\rho, z$, the expression for $\Delta W$ can be rewritten in the form $\Delta W=\Delta C(\rho, z) V^{2} / 2$ where $\Delta C(\rho, z)$, the change in the tip-electrode capacity caused by the presence of a nano-structure at the point $(\rho, z)$, is the final goal of our calculations.

We note that $\Delta C$ is a deviation from the capacity of a uniform sample which formally diverges ${ }^{2}$ but in fact is large but finite and determined by the macroscopic sample geometry. To remove this constant component containing no information on microscopic characteristics, various differential methods are used. For in-plane uniform samples (see Ref. 2 and Sec. II of the present work) the voltage derivative $d C / d V$ is usually used. As was shown in Sec. III, a similar procedure is also required for an array of nanowires connected to a conducting substrate. In the case of single nanostructures this is not necessary and by measuring spatial variations of capacity we can directly determine $\Delta C(\rho, z)$, as was done, e.g., in Ref. 11, where the capacitance of nanodots was obtained by subtracting a large stray capacitance from the experimentally measured value. The explanation is related to the fact that the above-mentioned nanostructures were recharged by the applied voltage $V$ and slowly decreasing Coulomb potentials caused an infinitely large contribution of distant elements to the capacity. For isolated nanodots and nanowires, $V$ causes only a short-range dipole polarization and the contribution of distant elements is irrelevant. For this reason, we can restrict further calculations to a single nanowire or nanodot although real structures often represent composites with a large concentration of these objects. 
It is necessary to note that the sensitivity of the method can be essentially enhanced if the probe tip is placed not outside the dielectric matrix but embedded into it. In this case the electric field around nanoparticles, Eq. (11), no longer contains the small factor $1 / \varepsilon$ so that for a given $V$ the energy of polarization $\Delta W$ and, hence, $\Delta C$ increase by the factor $\varepsilon^{2}$.

We want also to highlight that this section, contrary to Secs. II and III does not assume the presence of carriers in nanostructures and hence can be applied to any heterogeneous systems with different dielectric constants $\varepsilon$ of components, especially to ferroelectric-paraelectric blends with very high consrast in $\varepsilon$.

\section{B. Polarization and SCM of nanodots}

Let us find the dipole moment $\mathbf{p}$ acquired by a spherical nanodot of radius $w$ in the external electric field $\mathbf{E}$. The solution of this problem for a dielectric sphere with dielectric constant $\varepsilon_{i}$ differing from that of the matrix $\varepsilon$ is well known ${ }^{13}$ and we generalize it to the case of a doped nanodot with carrier density $n_{0}$. Due to a very small size $w$, the voltage drop on a dot is less than $k T / e$ even at high $V$, and we can consider the processes of screening in the linear approximation and find the potential profile $\varphi_{i}(r, \theta)$ in it ( $\theta$ is measured from the direction of $\mathbf{E}$ ) by solving the linear equation $\Delta \varphi_{i}=\lambda^{2} \varphi_{i}$ where $\lambda^{2}=4 \pi e^{2} n_{0} / k T$ (for simplicity we consider not very small dots and neglect quantization of their energy spectrum). For a dot with the center at $r=0$ the solution for $\varphi_{i}(r, \theta)$ should be matched at $r=w$ with the potential outside nanodot $\varphi_{e}(r, \theta)$ satisfying the Laplace equation $\Delta \varphi_{e}=0$ at $r>w$ and tending to the potential of external field $-E r \cos \theta$ at large $r$. The final answer for $\varphi_{e}$ is

$$
\begin{aligned}
& \varphi_{e}(r, \theta)=-E r \cos \theta-\frac{E w^{3}}{r^{2}} \alpha(\lambda a) \cos \theta, \\
& \alpha(x)=\frac{\varepsilon \mathrm{I}_{3 / 2}(x)-\varepsilon_{i}\left[x \mathrm{I}_{1 / 2}(x)-2 \mathrm{I}_{3 / 2}(x)\right]}{2 \varepsilon \mathrm{I}_{3 / 2}(x)+\varepsilon_{i}\left[x \mathrm{I}_{1 / 2}(x)-2 \mathrm{I}_{3 / 2}(x)\right]}
\end{aligned}
$$

$\left[\mathrm{I}_{n}(x)\right.$ is the Bessel function of imaginary argument].

The second term in $\varphi_{e}(r, \theta)$ has the form of a dipole potential so that we can claim the dipole moment $\mathbf{p}$ acquired by a dot in the external electric field is $\mathbf{p}=\alpha \mathbf{E}$. Using asymptotics of $\mathrm{I}_{n}(x)$, one can easily show that the polarizability $\alpha$ in the limits $\lambda w \rightarrow 0$ and $\lambda w \rightarrow \infty$ tends to known values $w^{3}\left(\varepsilon_{i}\right.$ $-\varepsilon) /\left(2 \varepsilon+\varepsilon_{i}\right)$ and $w^{3}$ characterizing, respectively, dielectrics and metals. Note that at $\varepsilon>\varepsilon_{i}$ there exists some critical value of $\lambda w$ for which the right-hand side of Eq. (12) vanishes, which means that dots with some diameter (or some doping level) will be "invisible" by SCM.

To analyze the spatial resolution of nanodot SCM, we calculate the radius $\Delta \rho$ at which the tip electric field given by Eqs. (11) drops twofold as compared with that at $\rho=0$ for the same values of $d, R$, and $z . \Delta \rho$ characterizes the distance from the nanodot at which the measured $\Delta C$ decreases by the factor of 4 in the course of in-plane scanning or, in other words, is a measure of the observed SCM image. The results in Fig. 2(a) show the decrease of an apparent dot size upon
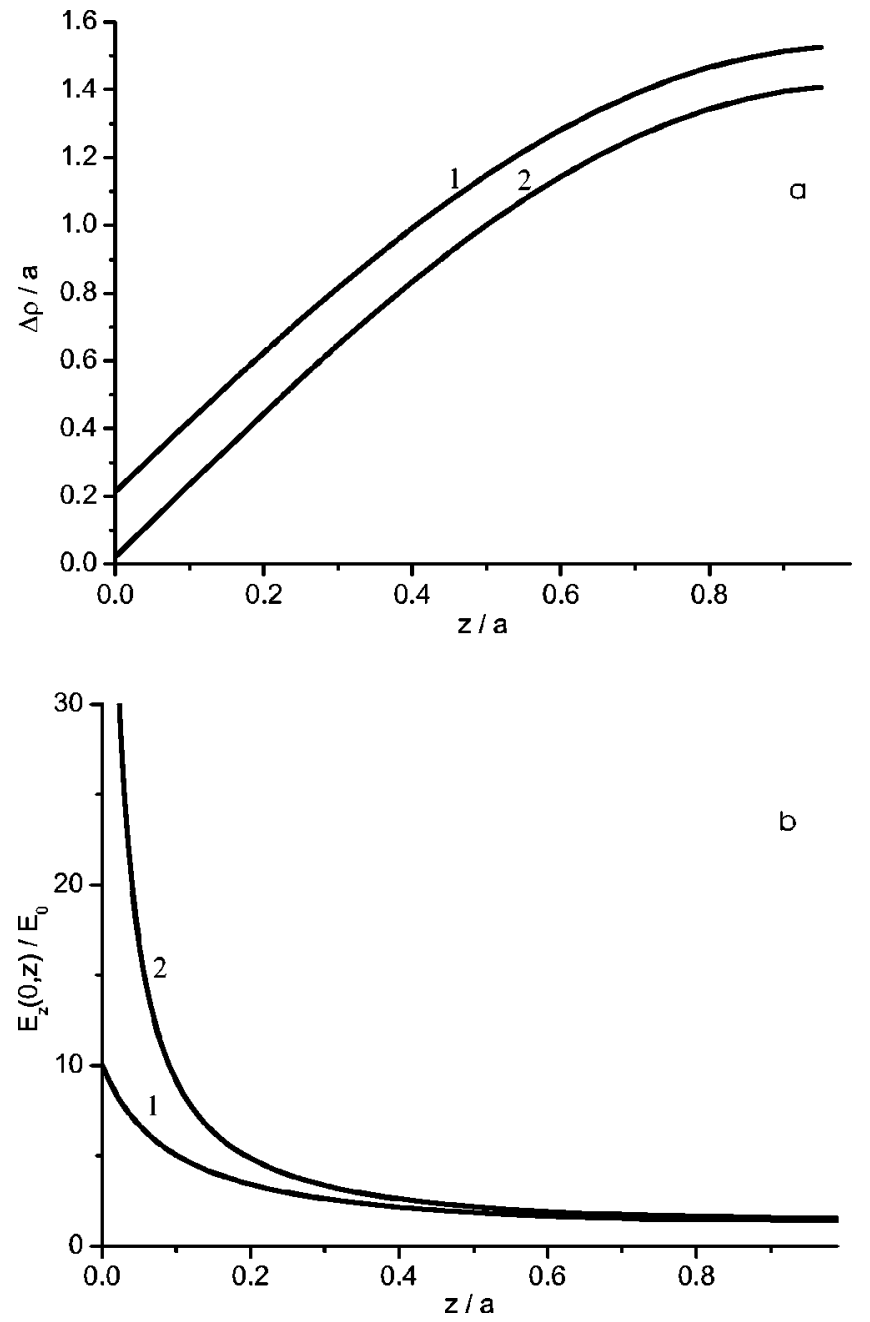

FIG. 2. Spatial resolution (a) and maximal electric field (b) at different depths for $\sqrt{d^{2}+R d}=0.1 a$ (1) and $\sqrt{d^{2}+R d}=0.01 a$ (2). The characteristic electric field $E_{0}=2 V /\{\varepsilon a \ln [(\sqrt{d+R}+\sqrt{d}) /$ $(\sqrt{d+R}-\sqrt{d})]\}$.

moving away from the surface for two different tips (or different sample thicknesses). In parallel, Fig. 2(b) shows the simultaneous decrease of $E_{z}(0, z)$ determining the measured value of $\Delta C$.

Equations (12) followed by conclusions related to spatial resolution of SCM assume the electric field in the vicinity of nanodots is uniform. This assumption may be violated in the closest vicinity and for small radius of a tip - that is, at very small $d, R$, and $z$. It means that the results shown in Fig. 2 remain adequate until the corresponding $\Delta \rho$ exceeds the nanodot radius $w$. If $\Delta \rho<w$, then the size of the SCM image is determined by $a$, rather than $\Delta \rho$-in other words, reproduces the real size of a nanodot.

Estimates show that, e.g., for the tip parameters $d=R$ $=5 \mathrm{~nm}$, nanodots with $w=10 \mathrm{~nm}$ placed near the sample surface give $\Delta C \sim 10^{-17} \mathrm{~F}$, which can be easily measured by the SCM equipment. ${ }^{1}$ The results of direct experimental SCM measurements of $\mathrm{Si}$ nanocrystals in $\mathrm{SiO}_{2}$ have been recently published in Ref. 12. 


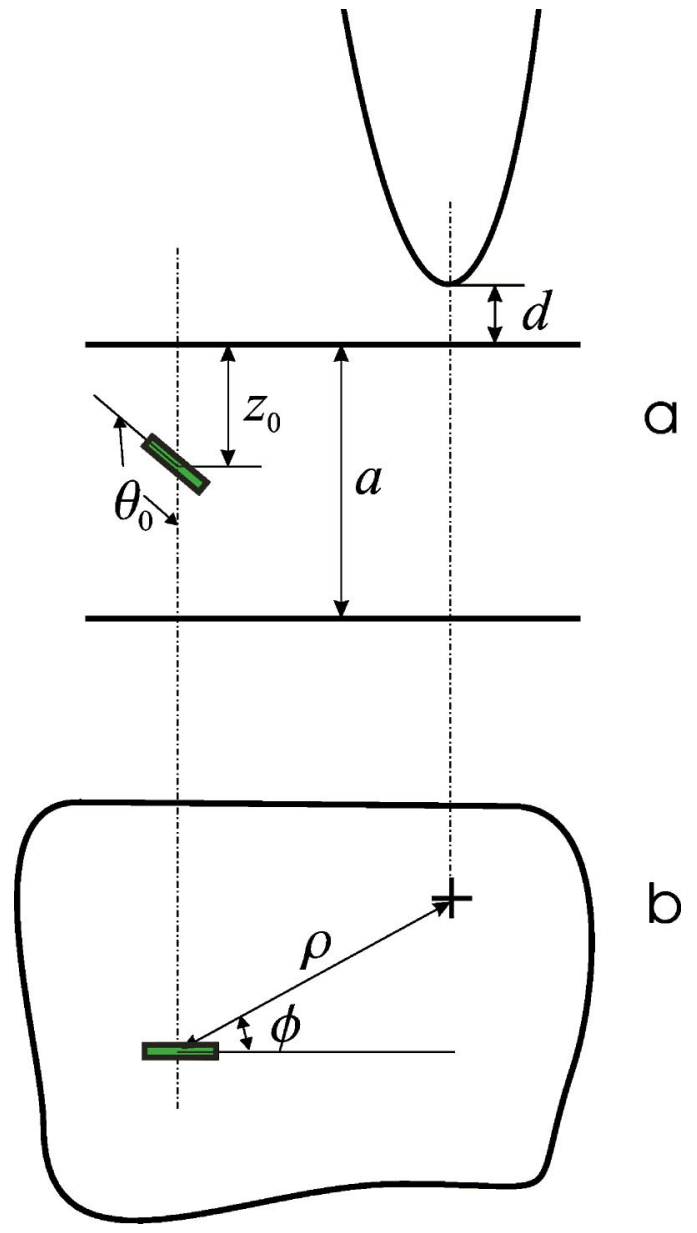

FIG. 3. Side view (a) and view from above (b) of SCM measurements of a single nanowire. The cross in (b) marks the in-plane position of the tip.

\section{Polarization and SCM of nanowires}

Consider polarization of a single nanowire. Contrary to the case of nanodots, the polarizability is strongly anisotropic and can be estimated from Ref. 13 for polarization of a dielectric and metallic ellipsoids. For $\mathbf{E}$ perpendicular to the wire axis, $\alpha_{\perp}=L w^{2}\left(\varepsilon_{i}-\varepsilon\right) /\left[2\left(\varepsilon_{i}+\varepsilon\right)\right]$ at $w \lambda \ll 1$ and $\alpha_{\perp}$ $=L w^{2}$ at $w \lambda \gg 1$ where $w$ and $L$ are, respectively, the radius and length of a wire. For semiconducting (and, of course, metallic) nanowires, $L$ definitely exceeds the screening length $\lambda^{-1}$ and the parallel component of polarizability corresponds to that of a metallic wire: $\alpha_{\|}=L^{3} /[16 \ln (L / w)]$.

For this reason, the visibility of nanowires depends on their orientation related to a local value of electric field. To analyze this problem in detail, assume a nanowire with the center at $\rho=0, z=z_{0}$ and axis tilted by angle $\theta_{0}$ from the $z$ axis. The tip position will be characterized by the fixed parameter $d$, as before, and by the polar coordinates $\rho, \phi$ where $\phi$ is measured from the $\rho$ projection of the nanowire axis (at $\theta_{0}=0$ when this projection is absent, the answer is $\phi$ independent). This geometry is shown in Fig. 3.

To calculate the SCM image for such nanowire, we note that its dipole moment, due to the strong anysotropy $\left(\alpha_{\perp}\right.$ $\left.\ll \alpha_{\|}\right)$, will be caused almost exclusively by the component

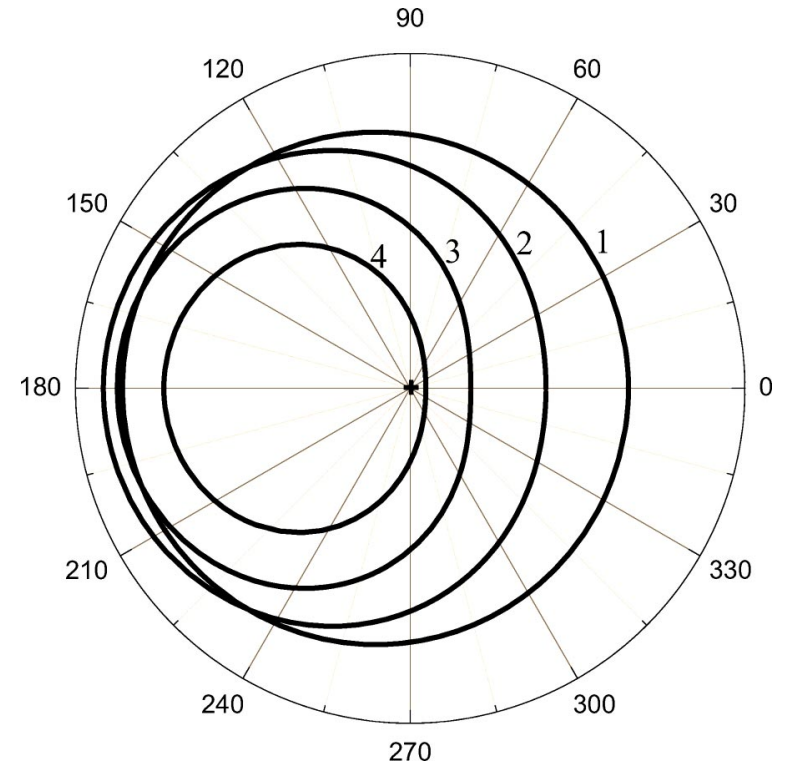

FIG. 4. Lines of equal $\Delta C$ around a single small nanowire (placed in the origin) with the tilt angle $\theta_{0}=10^{\circ}$ (curve 1 ), $30^{\circ}$ (curve 2), $50^{\circ}$ (curve 3 ), and $70^{\circ}$ (curve 4) for the case $\sqrt{d^{2}+R d}$ $=0.01 a, z_{0}=-0.1 a$. For each case, only one line corresponding to the electric field $E_{\|}=V / a$ is shown.

of electric field parallel to the wire axis, $E_{\|}$, so that, for the given voltage $V$, the measured value of $\Delta C \sim E_{\|}^{2}$. From Fig. 3 one can easily see that $E_{\|}=E_{z}\left(\rho, z_{0}\right) \cos \theta_{0}$ - $E_{\rho}\left(\rho, z_{0}\right) \sin \theta_{0} \cos \phi$ where $E_{z}$ and $E_{\rho}$ are given by Eqs. (11). Figure 4 illustrates the shape of iso- $E_{\|}$lines, connecting the points of equal $\Delta C$, around one nanowire. For a tilted nanowire, the lines are seen to be strongly distorted, mostly by shifting the center of gravity. This is due to the fact that even for the same distance $\rho$, the angle between the electric field and the nanowire axis and, hence, $E_{\|}$depend on $\phi$. For instance, in the geometry of Fig. 3 the mentioned angle will be less and, respectively, $\Delta C$ will be larger for the tip placed left of the nanowire than at the same distance right of it.

Figure 4 can make a wrong impression that the image of a tilted nanowire has almost circular character though noticeably shifted from the real position of a nanowire since in our approximate calculations we assumed the nanowire length $L$ to be much less than the characteristic length determining spatial variations of electric field (which is $\sim a$, except for the immediate vicinity of the tip). For longer wires, with $L$ comparable to $a$, one cannot characterize these by a single parameter $\alpha$ but should consider the spatial distribution of an induced charge along the wire and then express the measurable characteristic $\Delta C$ in terms of this distribution. The first problem can be solved if we recall that the potential created by a charged wire at its surface is approximately proportional to its linear charge density $\nu: \varphi_{1}(\xi) \simeq[2 \nu(\xi) / \varepsilon] \ln (L / w)$ where $\xi$ is the coordinate along the wire. ${ }^{10}$ Thus, an isolated conducting nanowire will remain equipotential in some external potential $\varphi$ (which means $\varphi+\varphi_{1}=$ const) if the charge density induced in it, $\nu(\xi)=\varepsilon[\varphi(\xi)-\bar{\varphi}] /[2 \ln (L / w)]$, where $\bar{\varphi}$ is the average potential along the nanowire. Since the change in electrostatic energy caused by the nanowire polarization, 


$$
\Delta W=\frac{1}{2} \int_{0}^{L} \nu(\xi) \varphi(\xi) d \xi=\frac{\varepsilon\left[\overline{\varphi^{2}}-(\bar{\varphi})^{2}\right]}{4 \ln (L / w)},
$$

the measured $\Delta C$ is also proportional to this characteristic.

Equations (13) and (17) can be used to calculate the capacitance pattern around a long nanowire with $L=a / 2$. Figure 5(a) shows the profile of $\Delta C$ along the axis $(\phi$ $\left.=0^{\circ}, 180^{\circ}\right)$ of a horizontal $\left(\theta_{0}=90^{\circ}\right)$ nanowire for two different distances from the surface $z_{0}$. The most peculiar feature of the figure is an essentially nonuniform SCM image of a long uniform nanowire, having maximal $\Delta C$ near its ends. This is due to the fact that the tip placed above the midpoint of a horizontal nanowire induces no dipole but only higherorder electric moments in it, while tip deviation towards any end increases polarization and, hence, the observed $\Delta C$. As in the case of nanodots (Sec. IV B), the amplitude of $\Delta C$ drops with $\left|z_{0}\right|$ since the local elecrtic field causing polarization of the nanostructure is high only in the vicinity of the tip. The last factor dramatically disturbs the image even for a small deviation from the horizontal nanowire orientation. Figures 5(b) and 5(c) show and that the maximum corresponding to the outermost nanowire end is dramatically suppresssed disappearing completely at the deviations exceeding $20^{\circ}\left(\theta_{0}<70^{\circ}\right)$.

\section{CONCLUSION}

We have considered application of SCM for the investigation of quantum wells, wires, and dots with characteristic sizes less than the electron screening length determining SCM spatial resolution in bulk samples. In this case, the observed SCM image does not reproduce the geometric characteristics of the system though it can be used for extracting some important information. The analysis of this problem cannot be performed using the approach developed in Ref. 2 for macroscopic samples and actually represents a new physical problem to be solved in different ways for different types of nanostructures. For quantum wells and nanowire arrays, SCM measurements give an opportunity to determine the doping level of these nanostructures. For individual nanowires and nanodots, SCM measurements do not allow one to get the real geometric size of nanostructures but the positions of SCM maxima give the in-plane location of the objects, the amplitude of $\Delta C$ variations gives information on their depth, and the pattern shape is extremely sensitive to the nanowire orientation.

\section{APPENDIX: ELECTRIC FIELD PROFILE BETWEEN THE TIP AND CONDUCTING PLANE}

The profile of an electric field existing in the absence of embedded nanostructures between the tip and conducting plane (the conducting substrate in Sec. III and the backside electrode in Sec. IV B) is calculated. The system has the same geometry as shown in Fig. 1(a) and, due to the boundary condition $\varphi(\rho,-a)=0$, the corresponding Laplace equation is the same as that of Sec. II in the linear regime $|V|$ $<V_{0}$. However, the results of Sec. II can not be directly applied here. The thickness of real samples determining the
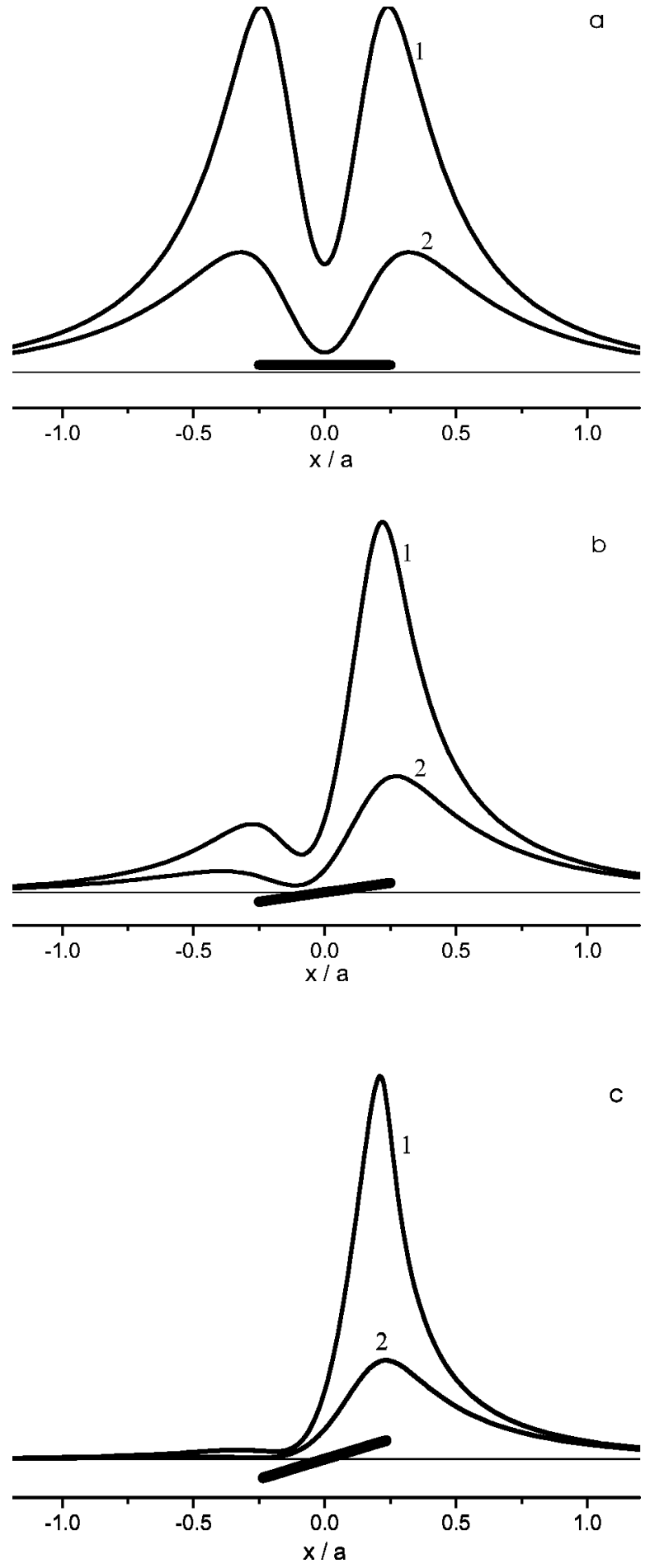

FIG. 5. Spatial dependence of $\Delta C$ (relative units) for the SCM tip with $\sqrt{d^{2}+R d}=0.01 a$ along the axis of a nanowire with tilt angle: (a) $\theta_{0}=90^{\circ}$ (horizontal nanowire), (b) $\theta_{0}=80^{\circ}$, and (c) $\theta_{0}$ $=70^{\circ}$ and the position of the center $z_{0}=-0.1 a$ (curves 1 ) and $z_{0}$ $=-0.2 a$ (curves 2 ). The dark segment shows the nanowire orientation in the vertical plane.

position of the backside electrode $a$ is sufficiently high to exceed the tip parameters $d$ and $R$, such that the small $a$ approximation used in Sec. II is definitely inadequate, and an exact solution of the Laplace equation at $-a \leqslant z \leqslant 0$ is re- 
quired. For the boundary condition $\varphi(\rho,-a)=0$ its general form is

$$
\varphi(\rho, z)=\int_{0}^{\infty} A(\lambda) \mathrm{J}_{0}(\lambda \rho) \sinh [\lambda(z+a)] d \lambda .
$$

Using Eq. (A1), we may connect $\varphi(\rho, z)$ with the electric field in vacuum near the interface:

$$
\begin{aligned}
\varphi(\rho, z)= & \frac{1}{\varepsilon} \int_{0}^{\infty} \frac{\sinh [\lambda(z+a)]}{\cosh (\lambda a)} \mathrm{J}_{0}(\lambda \rho) \\
& \times \int_{0}^{\infty} \rho^{\prime} \frac{\partial \varphi}{\partial z}\left(\rho^{\prime}, 0^{+}\right) \mathrm{J}_{0}\left(\lambda \rho^{\prime}\right) d \rho^{\prime} d \lambda .
\end{aligned}
$$

With the relationship between $\varphi(\rho, 0)$ and $(\partial \varphi / \partial z)\left(\rho, 0^{+}\right)$at the surface derived in Ref. 2 for a parabolic tip, we obtain the integral equation for the potential:

$$
\begin{aligned}
\varphi(\rho, z)= & \frac{2}{\varepsilon \ln \left(\frac{\sqrt{d+R}+\sqrt{d}}{\sqrt{d+R}-\sqrt{d}}\right)} \int_{0}^{\infty} \frac{\sinh [\lambda(z+a)]}{\cosh (\lambda a)} \mathrm{J}_{0}(\lambda \rho) \\
& \times \int_{0}^{\infty} \rho^{\prime} \frac{\left[V-\varphi\left(\rho^{\prime}, 0\right)\right]}{\sqrt{\left(\rho^{\prime}\right)^{2}+d^{2}+R d}}\left(\rho^{\prime}, 0^{+}\right) \mathrm{J}_{0}\left(\lambda \rho^{\prime}\right) d \rho^{\prime} d \lambda .
\end{aligned}
$$

The dielectric constant $\varepsilon$ in most semiconductors exceeds 10 , which allows us to solve Eq. (A3) by perturbation expansion in $1 / \varepsilon$. In the first approximation, we neglect $\varphi\left(\rho^{\prime}, 0\right)$ on the right-hand side of equation. This gives

$$
\begin{aligned}
\varphi(\rho, z)= & \frac{2 V}{\varepsilon \ln \left(\frac{\sqrt{d+R}+\sqrt{d}}{\sqrt{d+R}-\sqrt{d}}\right)} \int_{0}^{\infty} \frac{\sinh [\lambda(z+a)]}{\lambda \cosh (\lambda a)} \mathrm{J}_{0}(\lambda \rho) \\
& \times \exp \left(-\lambda \sqrt{d^{2}+R d}\right) d \lambda .
\end{aligned}
$$

The applicability limits of Eq. (A4) can be easily found by comparison of $\varphi(\rho, 0)$ with $V$. The expression, Eq. (A4), decreases monotonically with $\rho$ and hence the condition $|\varphi(0,0)|<|V|$ will guarantee adequacy of our approximation. Since $\int_{0}^{\infty} \tanh t \exp (-x t) d t / t$ at $x \ll 1$ is approximately $\ln (1 / x)$, the above-mentioned condition is reduced to

$$
\varepsilon \ln \left(\frac{\sqrt{d+R}+\sqrt{d}}{\sqrt{d+R}-\sqrt{d}}\right) \gg 2 \ln \left(\frac{a}{\sqrt{d^{2}+R d}}\right)
$$

and is violated for very small tip-sample distances $d$ [this condition is sufficient but not necessary so that the real applicability region of Eq. (A4) can be wider].
${ }^{1}$ C. C. Williams, Annu. Rev. Mater. Sci. 29, 471 (1999).

${ }^{2}$ H. E. Ruda and A. Shik, Phys. Rev. B 67, 235309 (2003).

${ }^{3}$ S. Luryi, Appl. Phys. Lett. 52, 501 (1988).

${ }^{4}$ D. Routkevich, A. A. Tager, J. Haruyama, D. Almawlawi, M. Moskovits, and J. M. Xu, IEEE Trans. Electron Devices 43, 1646 (1996).

${ }^{5}$ X. Mei, M. Blumin, M. Sun, D. Kim, Z. H. Wu, H. E. Ruda, and Q. X. Guo, Appl. Phys. Lett. 82, 967 (2003).

${ }^{6}$ B. J. Ohlson, M. T. Björk, M. H. Magnusson, K. Deppert, L. Samuelson, and L. R. Wallenberg, Appl. Phys. Lett. 79, 3335 (2001).

${ }^{7}$ Z. H. Wu, X. Y. Mei, D. Kim, M. Blumin, and H. E. Ruda, Appl.
Phys. Lett. 81, 5177 (2002).

${ }^{8}$ A. A. Tager, J. M. Xu, and M. Moskovits, Phys. Rev. B 55, 4530 (1997).

${ }^{9}$ N. S. Averkiev and A. Shik, Phys. Rev. B 59, 3250 (1999).

${ }^{10}$ N. S. Averkiev and A. Shik, Semiconductors 30, 112 (1996).

${ }^{11}$ H. Yamamoto, T. Takahashi, and I. Kamiya, Appl. Phys. Lett. 77, 1994 (2000).

${ }^{12}$ S. Decossas, F. Mazen, T. Baron, A. Souifi, and G. Bremond, Physica E (Amsterdam) 17, 543 (2003).

${ }^{13}$ L. D. Landau and E. M. Lifshits, Electrodynamics of Continuous Media (Pergamon, New York, 1984). 